\title{
Gas-filled Cavities in the Liver
}

\author{
Kazuyuki Hamada and Yasutsuna Sasaki
}

Key words: clostridium perfringens, septic shock, liver abscess, gastric cancer

(Intern Med 57: 2277, 2018)

(DOI: 10.2169/internalmedicine.0172-17)

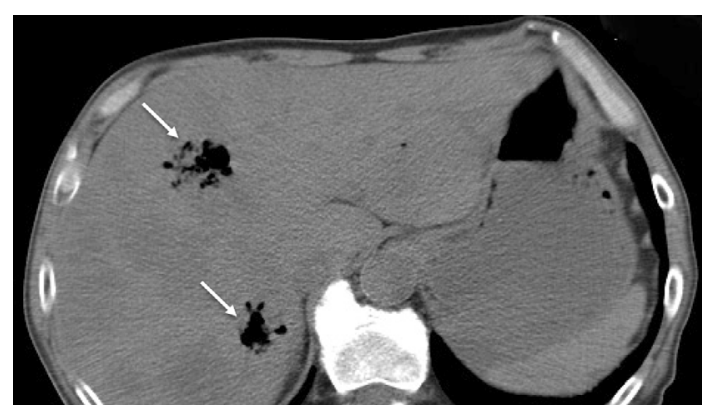

Picture 1.

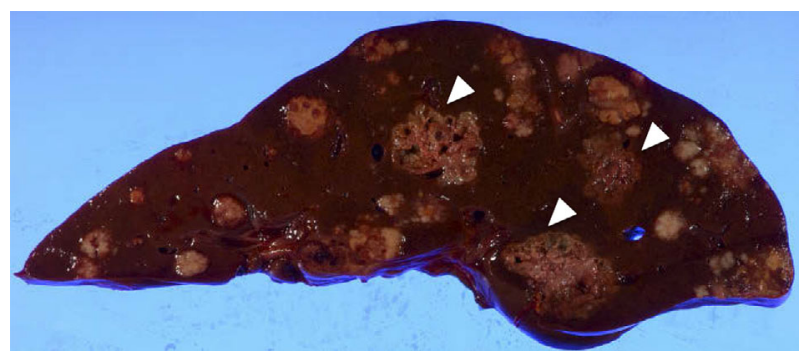

Picture 3.

A 68-year-old man receiving capecitabine and oxaliplatin for the treatment of metastatic gastric cancer presented with chills and shortness of breath continuing for 2 hours. On admission, he had a temperature of $37.4^{\circ} \mathrm{C}$, a blood pressure of $68 / 43 \mathrm{mmHg}$, and jaundice in the ocular conjunctiva. Blood tests revealed leukocytosis and increased levels of Creactive protein, procalcitonin, total bilirubin, and lactate dehydrogenase. Computed tomography revealed multiple hepatic gas-filled cavities (arrows) in addition to cancer metastases (Picture 1, 2). He was thus diagnosed with septic shock and liver abscess. He was treated with doripenem,

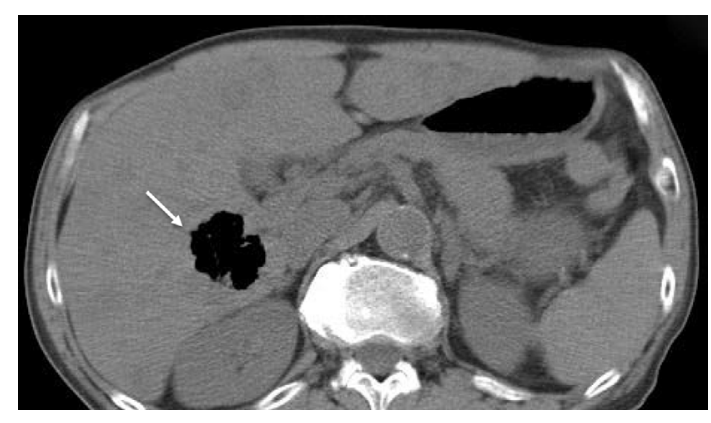

Picture 2.

azithromycin, and noradrenaline; however, 10 hours after hospitalization, he died due to progressive septic shock associated with acute hemolytic anemia. On autopsy, liver abscesses (arrowheads) were observed independent of the metastatic lesions (Picture 3). Blood cultures on admission and liver abscess lesions on autopsy tested positive for Clostridium perfringens. We diagnosed septic shock and liver abscess caused by $C$. perfringens. We suspect that the liver abscesses had formed through bacterial translocation caused by some mucous membrane disorder, which is a common side effect of capecitabine (1).

The authors state that they have no Conflict of Interest (COI).

\section{Reference}

1. Rolston KV. Infections in cancer patients with solid tumors. Infect Dis Ther 6: 69-83, 2017.

The Internal Medicine is an Open Access article distributed under the Creative Commons Attribution-NonCommercial-NoDerivatives 4.0 International License. To view the details of this license, please visit (https://creativecommons.org/licenses/ by-nc-nd/4.0/). 